\title{
Atypical Moyamoya Disease Associated with Midaortic Syndrome
}

\author{
Nishant Bhargava ${ }^{1, \odot ~ V i v e k ~ S i n g h ~}{ }^{2} \quad$ Chandradev Sahu ${ }^{3}$ \\ 1PDCC Neuroradiology, Department of Radiodiagnosis, Sanjay Gandhi \\ Postgraduate Institute of Medical Sciences, Lucknow, Uttar Pradesh, \\ India \\ 2Department of Radiodiagnosis, Sanjay Gandhi Postgraduate \\ Institute of Medical Sciences, Lucknow, Uttar Pradesh, India \\ ${ }^{3}$ Department of Radiodiagnosis, Pt. Jawahar Lal Nehru Memorial \\ Medical College, Raipur, Chhattisgarh, India
}

\author{
Address for correspondence Nishant Bhargava, MD, PDCC \\ Neuroradiology, Department of Radiodiagnosis, Sanjay Gandhi \\ Postgraduate Institute of Medical Sciences, New PMSSY Road, \\ Raibareli Road, Lucknow, Uttar Pradesh 226014, India \\ (e-mail: dr.nishantbhargava@gmail.com).
}

J Clin Interv Radiol ISVIR 2021;6:61-63.

\begin{abstract}
We report a case of a 4-year-old boy who presented with moyamoya disease associated with midaortic syndrome. He had been treated for severe persistent hyper-

Keywords

- moyamoya

- midaortic syndrome

- abdominal aorta tension until he suffered multiple episodes of seizure and cerebral ischemic attack. Cerebral angiography showed bilateral terminal internal carotid artery stenosis. Angiographic survey showed severe stenosis of abdominal aorta and bilateral proximal renal arteries. This is a very rare report of moyamoya disease with midaortic syndrome.
\end{abstract}

\section{Introduction}

Moyamoya disease is a rare cerebrovascular disorder characterized by progressive occlusion of the internal carotid artery (ICA) or its terminal branches, with formation of abnormal collateral vascular network called as "moyamoya vessels" at the base of the brain., Extracranial involvement is rare but documented in moyamoya disease. Most commonly involvement of renal arteries is seen, which can be a cause of persistent hypertension. However, to our knowledge, involvement of abdominal aorta with both renal arteries in the form of midaortic syndrome has not been reported in Indian subcontinent as a part of moyamoya disease spectrum.

Here we report an atypical case of moyamoya disease that underwent both cerebral and aortic angiography to evaluate a large territorial infarct and persistent hypertension.

\section{Case Report}

A 4-year-old male presented with chief complaints of three episodes of generalized tonic-clonic seizures since age of 18 months and left-sided hemiparesis for 1 month. On general examination the child was conscious, alert, and playful, with normally reacting bilateral pupils and normal visual

published online

April 29, 2021
DOI https://doi.org/

$10.1055 / \mathrm{s}-0041-1729465$

ISSN 2457-0214 acuity. Bilateral extraocular muscles showed full range of movements with no visual field defects. There was no facial asymmetry. All other cranial nerves were intact. On motor examination power was decreased in left upper and lower limbs. Deep tendon reflexes were adequate $(+2$ in all four limbs). Sensory system could not be adequately assessed.

He also had persistent hypertension despite antihypertensives.

Magnetic resonance imaging (MRI) of the brain showed a large area of diffusion restriction in right parieto-temporal lobe in middle cerebral artery (MCA) distribution ( - Fig. 1). Also, areas of T2/FLAIR-fluid attenuated inversion recoveryhyperintensity were seen in bilateral peri-ventricular regions, predominantly in bi-frontal lobes, most likely angiopathic changes. FLAIR image showed the typical "ivy sign" suggesting pial collaterals ( $\boldsymbol{- F i g . ~ 1 ) . ~ N o ~ f o c i ~ o f ~ b l o o m i n g ~ w e r e ~ s e e n . ~}$

On magnetic resonance angiogram (MRA), there was complete cutoff of right distal ICA in carotid fork region, proximal anterior cerebral artery (ACA), and MCA. Severe narrowing of left distal ICA was present. Also, focal narrowing of mid-basilar artery was seen. Multiple collaterals were noted from posterior circulation supplying the right MCA territory. MRA did not show any collateral in lenticulostriate or thalamostriate distribution ( - Fig. 2).

(C) 2021. Indian Society of Vascular and Interventional Radiology.

This is an open access article published by Thieme under the terms of the Creative Commons Attribution-NonDerivative-NonCommercial-License, permitting copying and reproduction so long as the original work is given appropriate credit. Contents may not be used for commercial purposes, or adapted, remixed, transformed or built upon. (https://creativecommons.org/licenses/by-nc-nd/4.0/).

Thieme Medical and Scientific Publishers Pvt. Ltd. A-12, 2nd Floor, Sector 2, Noida-201301 UP, India 


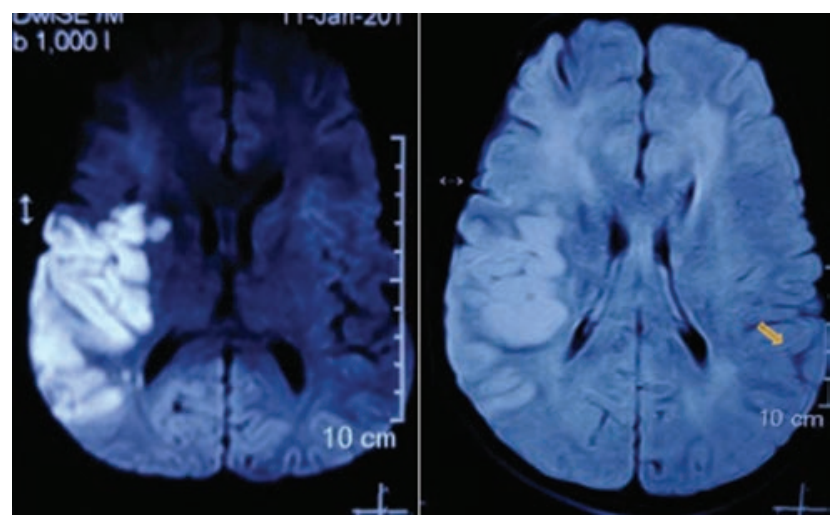

Fig. 1 Axial diffusion-weighted image showing a large area of diffusion restriction involving right MCA distribution. Axial fluid attenuated inversion recovery image shows gyral swelling in right parietal lobe with characteristic "ivy sign" (arrow).

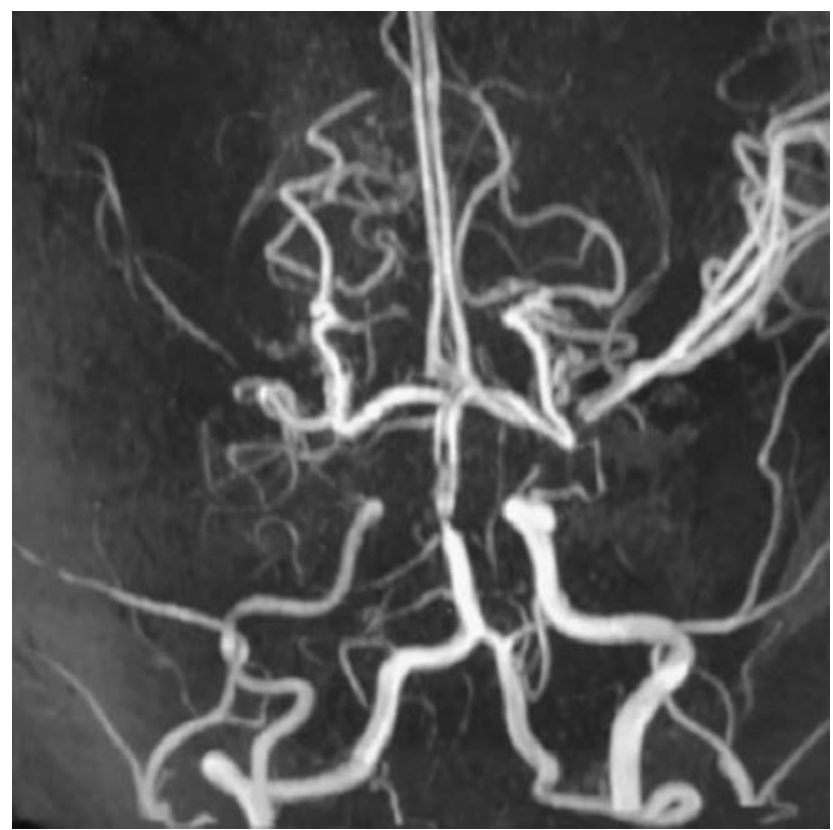

Fig. 2 Magnetic resonance angiogram showing abrupt cutoff of distal right internal carotid artery (ICA) with nonvisualization of anterior cerebral artery (ACA) and middle cerebral artery (MCA). Also, there is narrowing of left terminal left ICA, proximal ACA, and MCA. Focal narrowing of mid-basilar artery is seen. Collaterals are seen from posterior circulation supplying right MCA territory.

Vasculitis profile was negative. Another possibility of sickle cell disease/trait was considered; however, hemoglobin electrophoresis was negative for sickling. There was no history of any irradiation to head and neck. Coagulation profile was also normal. Erythrocyte sedimentation rate and C-reactive protein levels were within normal limits.

Subsequently, patient was sent for digital subtraction angiography, which showed right ICA terminating into ophthalmic artery with complete nonvisualization of distal right ICA, proximal ACA, and MCA (Suzuki stage V). Multiple small collaterals were seen in lenticulostriate distribution supplying ACA and MCA territory (moyamoya vessels). Also, few collaterals were seen from the dural branches of middle meningeal artery. Posterior circulation collaterals also supplied the right
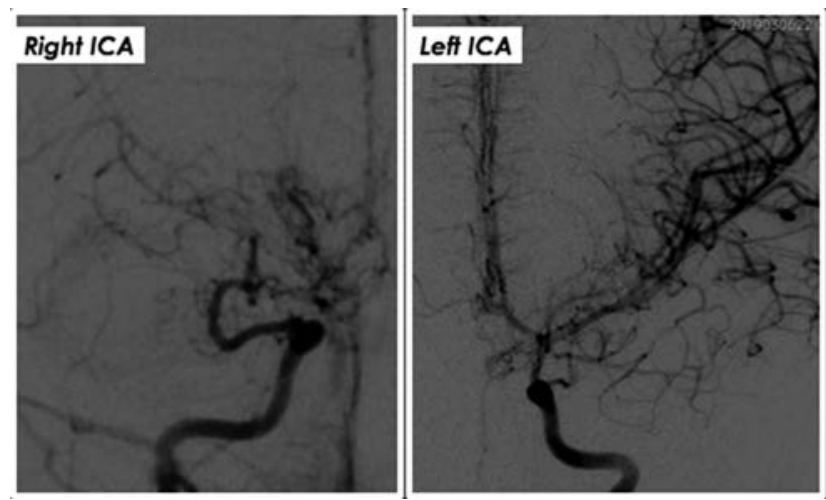

Fig. 3 Right internal carotid artery (ICA) angiogram showing termination of right ICA directly into ophthalmic artery. Anterior cerebral artery (ACA) and middle cerebral artery (MCA) are not visualized. Multiple small collaterals are seen in lenticulostriate distribution (puff of smoke). Left ICA angiogram showing severe narrowing of terminal ICA, proximal ACA, and MCA in carotid fork region. Few collaterals are seen in lenticulostriate distribution.

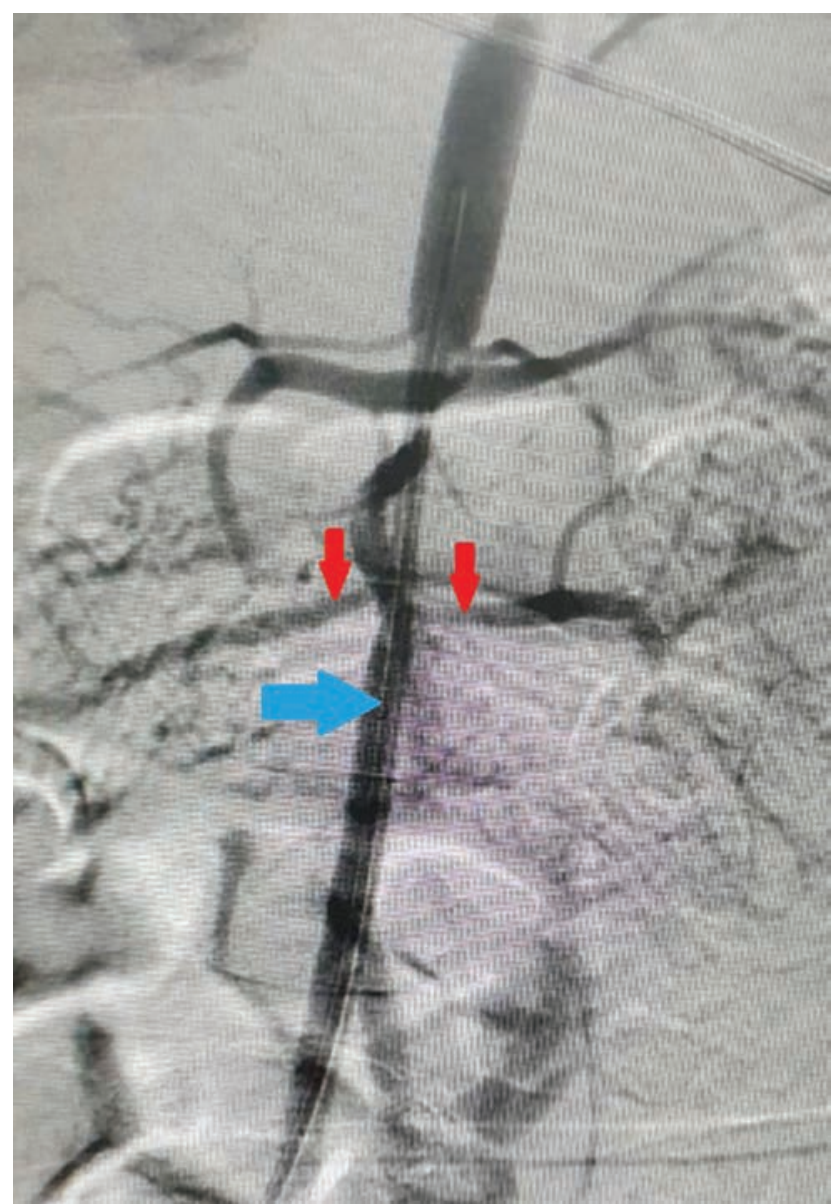

Fig. 4 Abdominal aortic angiogram shows severe narrowing of aorta (blue arrow) and proximal renal arteries (red arrows).

MCA distribution. Left ICA angiogram showed definite narrowing of distal ICA with few lenticulostriate collaterals and tenuous ACA and MCA (Suzuki stage IV; - Fig. $\mathbf{3}$ ).

Arch of aorta angiogram showed normal vascular anatomy. Abdominal aortic angiogram showed significant narrowing of aorta just proximal to origin of celiac trunk 
extending below the renal arteries. Also, there was proximal narrowing of bilateral renal arteries. Celiac trunk and superior mesenteric artery were normal ( - Fig. 4).

Right parietal craniotomy and superficial temporal artery-middle cerebral artery (STA-MCA) low-flow bypass with encephalomyodurosynangiosis were done to increase intracranial circulation in order to prevent future strokes. Post procedure, patient has same power as in preoperative state. Rest of the hospital stay remained uneventful and patient was discharged on day 10 of procedure on multiple antihypertensive medications to control blood pressure. On follow-up, patient remains stable with controlled blood pressure on multiple medications and no new ischemic episode.

\section{Discussion}

We described a 4-year-old boy with progressive moyamoya disease, displaying unusual stenosis of abdominal aorta and bilateral renal arteries.

Although extracranial involvement of moyamoya disease has been reported to a wide extent, mostly it has been limited to renal arteries with an approximate incidence of about $5 \%{ }^{3}$ A propensity to involve proximal renal arteries has been reported, which was consistent in our case too. Fibromuscular dysplasia (FMD) also involves the renal arteries; however, distal involvement with a characteristic string of beads appearance is reported. Also, FMD is seen in women of middle age group. ${ }^{1}$ Apart from renal arteries, involvement of other vessels including coronary, pulmonary, hepatic, splenic, superior mesenteric, and peripheral arteries is also seen in moyamoya disease, although involvement of other vessels of abdominal aorta is quite rare. ${ }^{3}$ An association with ACTA2 gene encoding smooth muscle actin has been linked with moyamoya disease, which might explain the involvement of renal arteries and descending aorta. ${ }^{14,5}$ Reports with midaortic syndrome in moyamoya disease are extremely rare. Midaortic syndrome is a rare vascular disease characterized by coarctation of the abdominal aorta and its visceral branches in children, often involving the renal arteries and causing severe renovascular hypertension. ${ }^{3,6} \mathrm{~A}$ common mechanism may underlie the pathogenesis of intracranial stenosis and midaortic syndrome in moyamoya disease. Pathologically it has been shown to be due to fibrous thickening of intima of the involved blood vessels. ${ }^{1,3}$ Korematsu et al believed existence of some systemic factors leading to vasculopathy in multiple vessels. ${ }^{3}$ In this particular case, there was no evidence of vasculitis/sickle cell disease/skull base tumor/irradiation/prothrombotic disorder. Atherosclerosis was out of question in a pediatric patient. No evidence of changes secondary to neurofibromatosis (café au lait spots, axillary freckling, plexiform neurofibroma, or any other intracranial changes) was seen. In the present case, patient symptoms were attributable to cerebral ischemia due to moyamoya disease, which has been demonstrated on angiogram, as stenosis of both supraclinoid internal carotid arteries, and on MRI, which shows large area of ischemic damage over right side. To prevent ischemia in moyamoya disease and increase intracranial circulation, many surgical procedures are performed including STA-MCA bypass, encephalomyosynangiosis, encephalomyodurosynangiosis, and encephalogaleosynangiosis. In the present case, STA-MCA low-flow bypass with encephalomyodurosynangiosis was done. Angioplasty and stenting of renal arteries in midaortic syndrome for uncontrolled blood pressure can be employed, although it was not needed in our case as blood pressure was controlled on multiple medications. Reported studies show poor long-term prognosis in patients with untreated midaortic syndrome with high risks of cardiac failure. Surgical treatment of midaortic syndrome has been reported with good results. ${ }^{7}$

\section{Conclusion}

We have presented a case of a 4-year-old boy with moyamoya disease accompanied with midaortic syndrome explaining the persistent hypertension. Therefore, we consider it necessary to evaluate aorta and renal arteries in patients with moyamoya spectrum presenting with hypertension.

\section{Conflict of Interest}

None declared.

\section{References}

1 Osamu T, Futoshi M, Takashi Y, et al. Prevalence of stenoocclusive lesions in the renal and abdominal arteries in moyamoya disease. AJR 2004;183:119-122

2 Uchikawa H, Fujii K, Fujita M, Okunushi T, Shimojo N. Atypical moyamoya syndrome with brain calcification and stenosis of abdominal aorta and renal arteries. Brain Dev 2017;39:710-713

3 Korematsu, et al. Moyamoya disease associated with midaortic syndrome. Pediatr Neurosurg 2007;43:54-59

4 Guey S, Tournier-Lasserve E, Hervé D, Kossorotoff M. Moyamoya disease and syndromes: from genetics to clinical management. Appl Clin Genet 2015;8:49-68

5 Milewicz DM, Østergaard JR, Ala-Kokko LM, et al. De novo ACTA2 mutation causes a novel syndrome of multisystemic smooth muscle dysfunction. Am J Med Genet A 2010; 152A(10):2437-2443

6 Graham LM, Zelenock GB, Erlandson EE, Coran AG, Lindenauer SM, Stanley JC. Abdominal aortic coarctation and segmental hypoplasia. Surgery 1979;86(4):519-529

7 O'Neill JA Jr, Berkowitz H, Fellows KJ, Harmon CM. Midaortic syndrome and hypertension in childhood. J Pediatr Surg 1995;30(2):164-171, discussion 171-172 